\title{
An Average-Case Sublinear Exact Li and Stephens Forward Algorithm
}

\author{
Yohei M. Rosen ${ }^{1}$ \\ University of California, Santa Cruz, California \\ New York University School of Medicine, New York, New York \\ yohei@ucsc.edu \\ (iD) https://orcid.org/0000-0002-3870-2169
}

\author{
Benedict J. Paten \\ University of California, Santa Cruz, California \\ bpaten@ucsc.edu
}

\begin{abstract}
Hidden Markov models of haplotype inheritance such as the Li and Stephens model allow for computationally tractable probability calculations using the forward algorithms as long as the representative reference panel used in the model is sufficiently small. Specifically, the monoploid $\mathrm{Li}$ and Stephens model and its variants are linear in reference panel size unless heuristic approximations are used. However, sequencing projects numbering in the thousands to hundreds of thousands of individuals are underway, and others numbering in the millions are anticipated.

To make the Li and Stephens forward algorithm for these datasets computationally tractable, we have created a numerically exact version of the algorithm with observed average case $\mathcal{O}\left(n k^{0.35}\right)$ runtime in number of genetic sites $n$ and reference panel size $k$. This avoids any tradeoff between runtime and model complexity. We demonstrate that our approach also provides a succinct data structure for general purpose haplotype data storage. We discuss generalizations of our algorithmic techniques to other hidden Markov models.
\end{abstract}

2012 ACM Subject Classification Theory of computation $\rightarrow$ Streaming, sublinear and near linear time algorithms, Applied computing $\rightarrow$ Bioinformatics

Keywords and phrases Haplotype, Hidden Markov Model, Forward Algorithm, Lazy Evaluation

Digital Object Identifier 10.4230/LIPIcs.WABI.2018.9

Related Version https://doi.org/10.1101/322396

Supplement Material https://github.com/yoheirosen/sublinear-Li-Stephens/

Funding This work was supported by the National Human Genome Research Institute of the National Institutes of Health under Award Number 5U54HG007990, the National Heart, Lung, and Blood Institute of the National Institutes of Health under Award Number 1U01HL137183-01, and grants from the W.M. Keck foundation and the Simons Foundation.

Acknowledgements We would like to thank Jordan Eizenga for his helpful discussions throughout the development of this work.

1 Yohei Rosen was supported in part by a Howard Hughes Medical Institute Medical Research Fellowship.

cc) (i) $\odot$ Yohei M. Rosen and Benedict J. Paten;

18th International Workshop on Algorithms in Bioinformatics (WABI 2018).

Editors: Laxmi Parida and Esko Ukkonen; Article No. 9; pp. 9:1-9:13

Leibniz International Proceedings in Informatics

LIPICS Schloss Dagstuhl - Leibniz-Zentrum für Informatik, Dagstuhl Publishing, Germany 


\section{Introduction}

Probabilistic models of haplotypes describe how variation is shared in a population. One application of these models is to calculate the probability $P(o \mid H)$ of a haplotype $o$ given the assumption that it is a member of a population represented by a reference panel of haplotypes $H$. This computation has been used in estimating recombination rates [8], a problem of interest in genetics and in medicine. It may also be used to detect errors in genotype calls.

Early approaches to haplotype modeling used coalescent [7] models which were accurate but computationally complex, especially when including recombination. Li and Stephens wrote the foundational computationally tractable haplotype model [8] with recombination. Under their model, the probability $P(o \mid H)$ can be calculated using the forward algorithm for hidden Markov models. Generalizations of their model have been used for haplotype phasing and genotype imputation. Most of these algorithms [10, 1, 14, 3, 12] use the forward probabilities calculated as intermediate values in the forward algorithm.

\subsection{The Li and Stephens model}

Consider a reference panel $H$ of $k$ haplotypes sampled from some population. Each haplotype $h_{j} \in H$ is a sequence $\left(h_{j, 1}, \ldots, h_{j, n}\right)$ of alleles at a contiguous sequence $1, \ldots, n$ of genetic sites. Classically [8], the sites are biallelic, but the model extends to multiallelic sites. [11]

Consider an observed sequence of alleles $o=\left(o_{1}, \ldots, o_{n}\right)$ representing another haplotype. The monoploid Li and Stephens model (LS) [8] specifies a probability that $o$ is descended from the population represented by $H$. LS can be written as a hidden Markov model wherein the haplotype $o$ is assembled by copying (with possible error) consecutive contiguous subsequences of haplotypes $h_{j} \in H$.

- Definition 1 ( $\mathrm{Li}$ and Stephens HMM). Define $x_{j, i}$ as the event that the allele $o_{i}$ at site $i$ of the haplotype $o$ was copied from the allele $h_{j, i}$ of haplotype $h_{j} \in H$. Take parameters

$$
\begin{aligned}
\rho_{i-1 \rightarrow i}^{*} & \text { the probability of any recombination between sites } i-1 \text { and } i \\
\mu_{i} & \text { the probability of a mutation from one allele to another at site } i
\end{aligned}
$$

and from them define the transition and recombination probabilities

$$
\begin{aligned}
& p\left(x_{j, i} \mid x_{j^{\prime}, i-1}\right)=\left\{\begin{array}{ll}
1-(k-1) \rho_{i} & \text { if } j=j^{\prime} \\
\rho_{i} & \text { if } j \neq j^{\prime}
\end{array} \quad \text { where } \rho_{i}=\frac{\rho_{i-1 \rightarrow i}^{*}}{k-1}\right. \\
& p\left(o_{i} \mid x_{j, i}\right)=\left\{\begin{array}{ll}
1-(A-1) \mu_{i} & \text { if } o_{i}=h_{j, i} \\
\mu_{i} & \text { if } o_{i} \neq h_{j, i}
\end{array} \quad \text { where } A=\right.\text { number of alleles }
\end{aligned}
$$

We will write $\mu_{i}(j)$ as shorthand for $p\left(o_{i} \mid x_{j, i}\right)$. We will also define the values of the initial probabilities $p\left(x_{j, 1}, o_{1} \mid H\right)=\frac{\mu_{1}(j)}{k}$.

Let $P(o \mid H)$ be the probability that haplotype $o$ was produced from population $H$. The forward algorithm for hidden Markov models allows calculation of this probability in $\mathcal{O}\left(n k^{2}\right)$ time using an $n \times k$ dynamic programming matrix of forward states

$$
p_{i}[j]=P\left(x_{j, i}, o_{1}, \ldots, o_{i} \mid H\right)
$$

In practice, the $\mathrm{Li}$ and Stephens forward algorithm is $\mathcal{O}(n k)$. (See $\S 3$ ) 


\subsubsection{Li and Stephens like algorithms for large populations}

The $\mathcal{O}(n k)$ time complexity of the forward algorithm is intractable for reference panels with large size $k$. The UK Biobank has amassed $k=500,000$ array samples. Whole genome sequencing projects, with a denser distribution of sites, are catching up. Major sequencing projects with $k=100,000$ or more samples are nearing completion. Others numbering $k$ in the millions have been announced. These large population datasets have significant potential benefits: They are statistically likely to more accurately represent population frequencies and those employing genome sequencing can provide phasing information for rare variants.

In order to handle datasets with size $k$ even fractions of these sizes, modern haplotype inference algorithms depend on models which are simpler than the Li and Stephens model or which sample subsets of the data. For example, the common tools Eagle-2, Beagle, HAPI-UR and Shapeit-2 and $-3[10,1,14,3,12]$ either restrict where recombination can occur, fail to model mutation, model long-range phasing approximately or sample subsets of the reference panel.

Lunter's "fastLS" algorithm [11] demonstrated that haplotypes models which include all $k$ reference panel haplotype could find the Viterbi maximum likelihood path in time sublinear in $k$, using preprocessing to reduce redundant information in the algorithm's input. However, his techniques do not extend to the forward and forward-backward algorithms.

\subsection{Our contributions}

We have developed an arithmetically exact forward algorithm whose expected time complexity is a function of the expected allele distribution of the reference panel. This expected time complexity proves to be $\mathcal{O}\left(k^{0.35}\right)$ in reference panel size. We have also developed a technique for succinctly representing large panels of haplotypes whose size also scales as a sublinear function of the expected allele distribution.

Our forward algorithm contains three optimizations, all of which might be generalized to other bioinformatics algorithms. In (§2), we rewrite the reference panel as a sparse matrix containing the minimum information necessary to directly infer all allele values. In (§3), we define recurrence relations which are numerically equivalent to the forward algorithm but use minimal arithmetic operations. In (§4), we delay computation of forward states using a lazy evaluation algorithm which benefits from blocks of common sequence. Our methods apply to other models which share certain properties with the monoploid Li and Stephens model.

\section{Sparse representation of haplotypes}

The forward algorithm to calculate the probability $P(o \mid H)$ takes as input a length $n$ vector $o$ and a $k \times n$ matrix of haplotypes $H$. Therefore time complexity better than $\mathcal{O}(n k)$ is impossible unless there is preprocessing of its input. However, such preprocessing can be amortized over many queries $o$.

\subsection{Information content of a reference panel}

Recall that $\left(o_{i}\right)_{i=1}^{n}$ is the allele sequence of the emitted haplotype $o$. (§3) will show that $\phi_{i}\left(o_{i}\right), 1 \leq i \leq n$ defined below are sufficient data to calculate $P(o \mid H)$.

- Definition 2. The information content $\phi$ of $H$ for allele $a$ at site $i$ is defined as

$$
\phi_{i}(a)=\left\{\begin{array}{lll}
\operatorname{Match}_{i}(a) & :=\left\{h_{j} \mid h_{j, i}=a\right\} & \text { if }\left|\operatorname{Match}_{i}(a)\right| \leq\left|\operatorname{NonMatch}_{i}(a)\right| \\
\operatorname{NonMatch}_{i}(a) & :=\left\{h_{j} \mid h_{j, i} \neq a\right\} & \text { if }\left|\operatorname{NonMatch}_{i}(a)\right|<\left|\operatorname{Match}_{i}(a)\right|
\end{array}\right.
$$




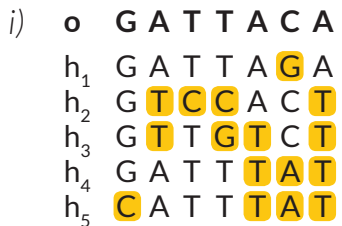

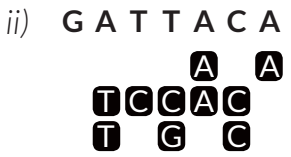

C iii) G A T T A C A

$h_{5} h_{2} h_{2} h_{2} h_{1} h_{2} h_{1}$ $h_{3} \quad h_{3} h_{2} h_{3}$

Figure 1 i) Reference panel $\left\{h_{1}, \ldots, h_{5}\right\}$ with mismatches to haplotype $o$ shown in yellow. ii) Alleles at site $i$ of elements of $\phi_{i}\left(o_{i}\right)$ in black. iii) Vectors to encode $\phi_{i}\left(o_{i}\right)$ at each site.

We will often abuse notation and refer to the $h_{j} \in \phi_{i}(a)$ by their indices $j$ alone.

\subsection{Relation of information content to allele frequency spectrum}

Our sparse representation of the haplotype reference panel benefits from the recent finding [6] that the distribution over sites of minor allele frequencies is biased towards low frequencies ${ }^{2}$.

We will compute the expected time sum of the information content over all sites assuming first that all sites are biallelic ${ }^{3}$. In the biallelic case $\phi_{i}(\cdot)$ is always the set of haplotypes displaying the minor allele at site $i$ and the distribution of $\phi_{i}(a)$ is the allele frequency spectrum.

- Lemma 3. Let $\mathbb{E}[\bar{f}](k)$ be the expected mean minor allele frequency for $k$ genotypes. Then

$$
\mathbb{E}\left[\frac{1}{n} \sum_{i=1}^{n}\left|\phi_{i}(a)\right|\right]=\mathbb{E}[\bar{f}](k)
$$

- Corollary 4. If $\mathcal{O}(\mathbb{E}[\bar{f}])<\mathcal{O}(k)$, then $\mathcal{O}\left(\sum_{i}\left|\phi_{i}(a)\right|\right)<\mathcal{O}(n k)$ in expected value.

\subsection{Implementation}

For biallelic sites, we store our $\phi_{i}$ 's using a length- $n$ vector of length $\left|\phi_{i}\right|$ vectors containing the indices $j$ of the haplotypes $h_{j} \in \phi_{i}$ and a length- $n$ vector listing the major allele at each site. (See Figure 1 panel iii) Random access by key $i$ to iterators to the first elements of sets $\phi_{i}(a)$ is $\mathcal{O}(1)$ and iteration across these $\phi_{i}(a)$ is linear in the size of $\phi_{i}(a)$. For multiallelic sites, the data structure uses slightly more space but has the same speed guarantees.

Generating these data structures takes $\mathcal{O}(n k)$ time but is embarrassingly parallel in $n$. Our "*.slls" data structure doubles as a succinct haplotype index which could be distributed instead of a large vcf record. A vcf $\rightarrow$ slls conversion tool is found in our github repository.

Adding or rewriting a haplotype is constant time per site per haplotype unless this edit changes which allele is the most frequent. This allows our algorithm to extend to uses of the $\mathrm{Li}$ and Stephens model where one might want to dynamically edit the reference panel.

\section{Efficient dynamic programming}

We begin with the recurrence relation of the $\mathcal{O}(n k) \mathrm{Li}$ and Stephens forward algorithm [8]. To establish our notation, recall that $p_{i}[j]=P\left(x_{j, i}, o_{1}, \ldots, o_{i} \mid H\right)$, that we write $\mu_{i}(j)$ as

2 We confirm these results in section 5.2

3 The generalization is trivial 
shorthand for $p\left(o_{i} \mid x_{j, i}\right)$ and that we have initialized $p_{1}[j]=p\left(x_{j, 1}, o_{1} \mid H\right)=\frac{\mu_{1}(j)}{k}$. For $i>1$, we may then write:

$$
\begin{aligned}
p_{i}[j] & =\mu_{i}(j)\left(\left(1-k \rho_{i}\right) p_{i-1}[j]+\rho_{i} S_{i-1}\right) \\
S_{i} & =\sum_{j=1}^{k} p_{i}[j]
\end{aligned}
$$

We will reduce the number of summands in (9) and reduce the number indices $j$ for which (8) is evaluated. This will use the information content defined in (§2.1).

- Lemma 5. The summation (9) is calculable using strictly fewer than $k$ summands.

Proof. Suppose first that $\mu_{i}(j)=\mu_{i}$ for all $j$. Then

$$
\begin{aligned}
S_{i}=\sum_{j=1}^{k} p_{i}[j] & =\mu_{i} \sum_{j=1}^{k}\left(\left(1-k \rho_{i}\right) p_{i-1}[j]+\rho_{i} S_{i-1}\right) \\
& =\mu_{i}\left(\left(1-k \rho_{i}\right) S_{i-1}+k \rho_{i} S_{i-1}\right)=\mu_{i} S_{i-1}
\end{aligned}
$$

Now suppose that $\mu_{i}(j)=1-\mu_{i}$ for some set of $j$. We must then correct for these $j$. This gives us

$$
S_{i}=\mu_{i} S_{i-1}+\frac{1-\mu_{i}-\mu_{i}}{1-\mu_{i}} \sum_{j \text { where } \mu_{i}(j) \neq \mu_{i}} p_{i}[j]
$$

The same argument holds when we reverse the roles of $\mu_{i}$ and $1-\mu_{i}$. Therefore we can choose which calculation to perform according to which involves a sum with fewer summands. This gives us the following formula:

$$
S_{i}=\alpha S_{i-1}+\beta \sum_{j \in \phi_{i}\left(o_{i}\right)} p_{i}[j]
$$

where

$$
\begin{array}{llrl}
\alpha=\mu_{i} & \beta=\frac{1-2 \mu_{i}}{1-\mu_{i}} & \text { if } \phi_{i}(a)=\operatorname{Match}_{i}(a) \\
\alpha=1-\mu_{i} & \beta=\frac{2 \mu_{i}-1}{\mu_{i}} & \text { if } \phi_{i}(a)=\operatorname{NonMatch}_{i}(a)
\end{array}
$$

Lemma 6. If $j \notin \phi_{i}\left(o_{i}\right)$ and $j \notin \phi_{i-1}\left(o_{i-1}\right)$, then $S_{i}$ can be calculated without knowing $p_{i-1}[j]$ and $p_{i}[j]$, as can $p_{i}\left[j^{\prime}\right]$ for $j^{\prime} \neq j$.

Proof. By inspection of equation (13).

- Corollary 7. The recurrences (9) and the minimum set of recurrences (8) needed to compute (9) can be evaluated in $\mathcal{O}(|\phi|)$ time, assuming that $p_{i-1}[j]$ have been computed $\forall j \in \phi_{i}\left(o_{i}\right)$.

We address the assumption on prior calculation of the necessary $p_{i-1}[j]$ 's in section 4 .

\subsection{Time complexity}

Recall that we defined $\mathbb{E}[\bar{f}](k)$ as the expected mean minor allele frequency in a sample of size $k$. By Corollary 7 the procedure in eq. (13) has expected time complexity $\mathcal{O}(n \mathbb{E}[\bar{f}](k))$. 


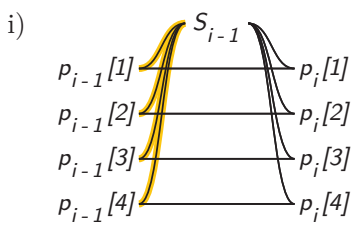

ii)

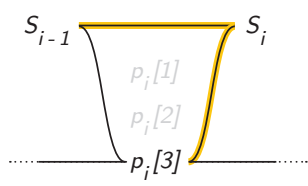

Figure 2 Using the example that at site $i, \phi_{i}\left(o_{i}\right)=\left\{h_{3}\right\}$, we illustrate the number of arithmetic operations used in i) the conventional $\mathcal{O}(n k) \mathrm{Li}$ and Stephens HMM recurrence relations ii) Our procedure specified in equation (13). Black lines correspond to arithmetic operations; operations which cannot be parallelized over $j$ are colored yellow.

\section{$4 \quad$ Lazy evaluation of dynamic programming rows}

Corollary 7 was conditioned on the assumption that specific forward probabilities had already been evaluated. We will describe a second algorithm which performs this task efficiently by avoiding arithmetic which will prove unnecessary at future steps. ${ }^{4}$

\subsection{Eliminating redundant recurrence evaluations}

The recurrence relations (8) are linear maps $r_{i}[j]: \mathbb{R} \rightarrow \mathbb{R}$ of the form

$$
r_{i}[j]: x_{j} \longmapsto \alpha_{i} x_{j}+\beta_{i}
$$

Let us formalize this notion of recurrence relation as linear map:

Definition 8. For any $i_{1}<i_{2}$, define the update map $r_{i_{1} \rightarrow i_{2}}[j]=r_{i_{2}}[j] \circ r_{i_{2}-1}[j] \circ \cdots \circ r_{i_{1}+1}[j]$

This update map is defined such that $r_{i_{1} \rightarrow i_{2}}[j]\left(p_{i_{1}}[j]\right)=p_{i_{2}}[j]$.

- Lemma 9. At each $i$ there exist only two unique maps among the $r_{i}[j]$.

Proof. Assume, without loss of generality, that $\phi_{i}(a)=\operatorname{Match}_{i}(a)$. Then define $\mu_{i}^{\circ}=\mu_{i}$ and $\mu_{i}^{\bullet}=1-\mu_{i}$. Then

$$
\begin{aligned}
& r_{i}^{\circ}(x)=\mu_{i}^{\circ}\left((1-k \rho) x+\rho S_{i}\right) \\
& r_{i}^{\bullet}(x)=\mu_{i}^{\bullet}\left((1-k \rho) x+\rho S_{i}\right)=\frac{\mu_{i}^{\bullet}}{\mu_{i}^{\circ}} r_{i}^{\circ}
\end{aligned}
$$

If $\phi_{i}(a) \neq \operatorname{Match}_{i}(a)$ then the same is true with the definitions of $\mu_{i}^{\circ}$ and $\mu_{i}^{\bullet}$ switched. This lemma allows us to rewrite each $r_{i_{1} \rightarrow i_{2}}[j]$ as a binary vector of the form $(\circ, \circ, \bullet, \ldots, \circ, \bullet, \circ)$.

We start with a sketch of the general concept of our algorithm

When Algorithm 1 is applied independently to all $h_{j}$, the aggregate algorithm has $\mathcal{O}(n k)$ time complexity, so we will share work between haplotypes $j$ using equivalence classes segregated by runs of homology.

\footnotetext{
4 This approach is known as lazy evaluation.
} 


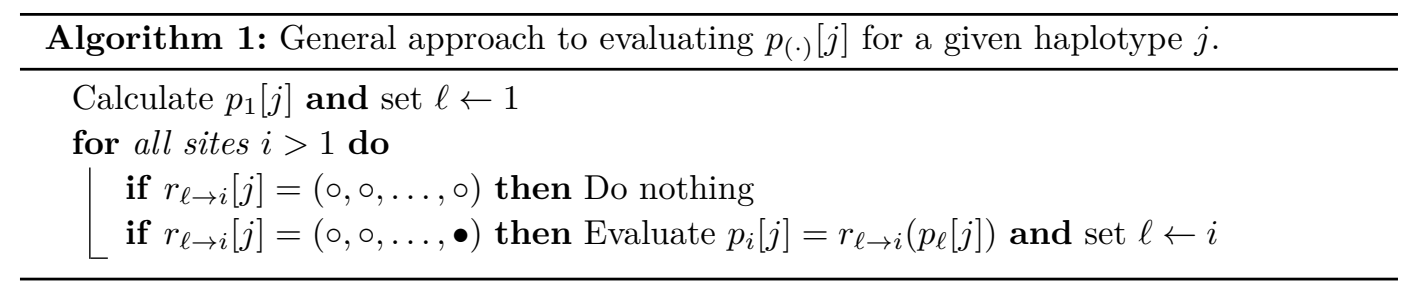

\subsection{Equivalence classes of update map prefixes}

Consider two different instances of the for loop in Algorithm 1, where the first is to evaluate $p_{(\cdot)}\left[j_{1}\right]$ and the second to evaluate $p_{(\cdot)}\left[j_{2}\right]$. Suppose that both are halted at the same step $i$, and suppose that at this step, the marker variable $\ell$ is the same for both of them. Then the sequences of $h_{j_{1}}$ and $h_{j_{2}}$ are identical between $\ell$ and $i$, and therefore

$$
r_{\ell \rightarrow i}\left[j_{1}\right]=r_{\ell \rightarrow i}\left[j_{2}\right]=\underbrace{(\circ, \circ, \ldots, \circ)}_{i-\ell}
$$

Therefore at each step $i$, we can divide the haplotype indices $j$ into equivalence classes $J[\ell]$ for which

1. The current marker variable $\ell$ is the same for all $j$ in this equivalence class

2. The map $r_{\ell \rightarrow i}[j]$ is the same for all $j$ in this equivalence class

And so we only need to calculate $r_{\ell \rightarrow i}[j]$ at most once per nonempty equivalence class $J[\ell]$, and for this map we write $r_{\ell \rightarrow i}$. The following lemma allows us to be even more efficient.

Lemma 10. If $i_{1}<i_{2}<i$, then $r_{i_{1} \rightarrow i}=r_{i_{2} \rightarrow i} \circ r_{i_{1} \rightarrow i_{2}}$

Lemma 10 allows us to calculate intermediate prefixes of the maps $r_{\ell \rightarrow i}$ and extend them at a later time. To make this concrete, suppose that we have an index $\pi_{\ell}$ ("prefix") where $\ell<\pi_{\ell}<i$. Then we can evaluate the prefix $r_{\ell \rightarrow \pi_{\ell}}$ of $r_{\ell \rightarrow i}$ knowing that $r_{\ell \rightarrow i}=r_{\pi_{\ell} \rightarrow i} \circ r_{\ell \rightarrow \pi_{\ell}}$ can be evaluated at a later time.

\subsection{The lazy evaluation algorithm}

Our full lazy evaluation algorithm stores the following state data at each step. The algorithm initialization is described in Algorithm 2 and the recurrence in Algorithm 3.

- The maps $j \mapsto J[\ell]$ from haplotype to its equivalence class; $\ell$ defined as the index at which $p_{(\cdot)}[j]$ was most recently calculated

- The maps $\ell \mapsto \pi_{\ell}$ mapping $\ell$ to the index $\pi_{\ell}$ for which a prefix $r_{\ell \rightarrow \pi_{\ell}}$ of $r_{\ell \rightarrow i}$ which was most recently calculated

- The maps $\ell \mapsto r_{\ell \rightarrow \pi_{\ell}}$ mapping $\ell$ to the the prefix $r_{\ell \rightarrow \pi_{\ell}}$ of $r_{\ell \rightarrow i}$ which was most recently calculated

- The maps $r_{1}^{\circ}, r_{2}^{\circ}, \ldots, r_{i}^{\circ}$ from which all maps $r_{(\cdot) \rightarrow(\cdot)}$ are formed

Calculating a closed form expression for the time complexity of the lazy evaluation algorithm 3 is not straightforward. It is easy to show that it bounded by $\mathcal{O}(n k)$, since the first loop is worst-case $\mathcal{O}(k)$. However, we find experimentally, this lazy evaluation component does not contribute to overall computational complexity. (See Fig. 6) 

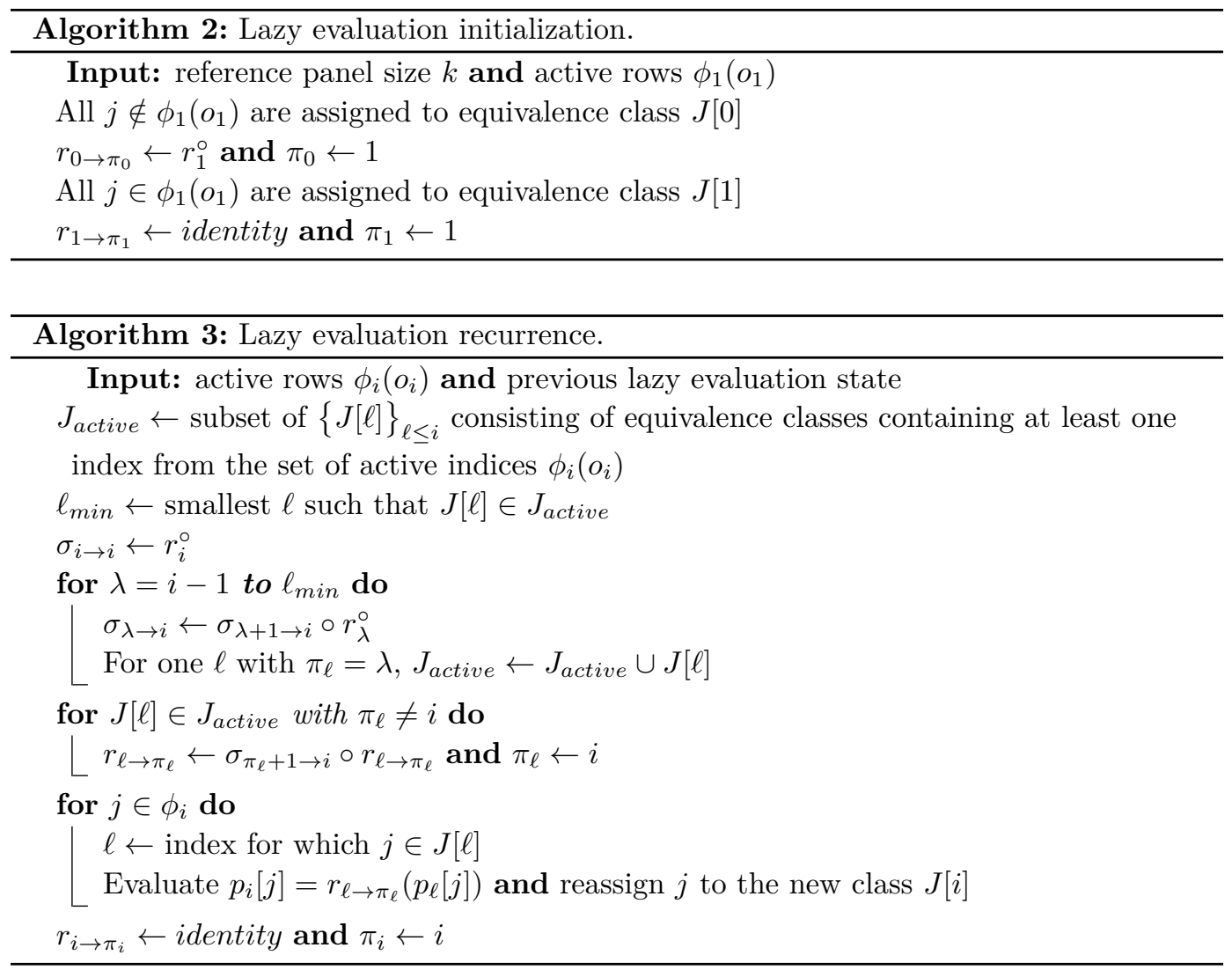

\section{Results}

\subsection{Implementation}

Our algorithm was implemented as a $\mathrm{C}++$ library located at https://github.com/yoheirosen/ sublinear-Li-Stephens Details of Algorithm 3 will be found there.

We also implemented the linear time monoploid Li and Stephens forward algorithm in $\mathrm{C}++$ as to evaluate it on identical footing. Profiling was performed using a single Intel Xeon X7560 core running at $2.3 \mathrm{GHz}$ on a shared memory machine. Our reference panels $H$ were the phased haplotypes from the 1000 Genomes [2] phase 3 vcf records for chromosome 22 and subsamples thereof. Haplotypes $o$ were randomly generated simulated descendants.

\subsection{Minor allele frequency distribution for the 1000 Genomes dataset}

We simulated haplotypes $o$ of 1,000,000 bp length on chromosome 22 and recorded the sizes of the sets $\phi_{i}\left(o_{i}\right)$ for $k=5008$. These data produced a mean $\left|\phi_{i}\left(o_{i}\right)\right|$ of 59.9 , which is $1.2 \%$ of the size of $k$. We have plotted the distribution of $\left|\phi_{i}\left(o_{i}\right)\right|$ which we observed from this experiment in (Fig. 4). It is skewed toward low frequencies; the minor allele is unique at $71 \%$ of sites, and it is below $1 \%$ frequency at $92 \%$ of sites.

\subsection{Comparison of our algorithm with the linear time forward algorithm}

In order to compare the dependence of our algorithm's runtime on haplotype panel size $k$ against that of the standard linear LS forward algorithm, we measured the CPU time per 


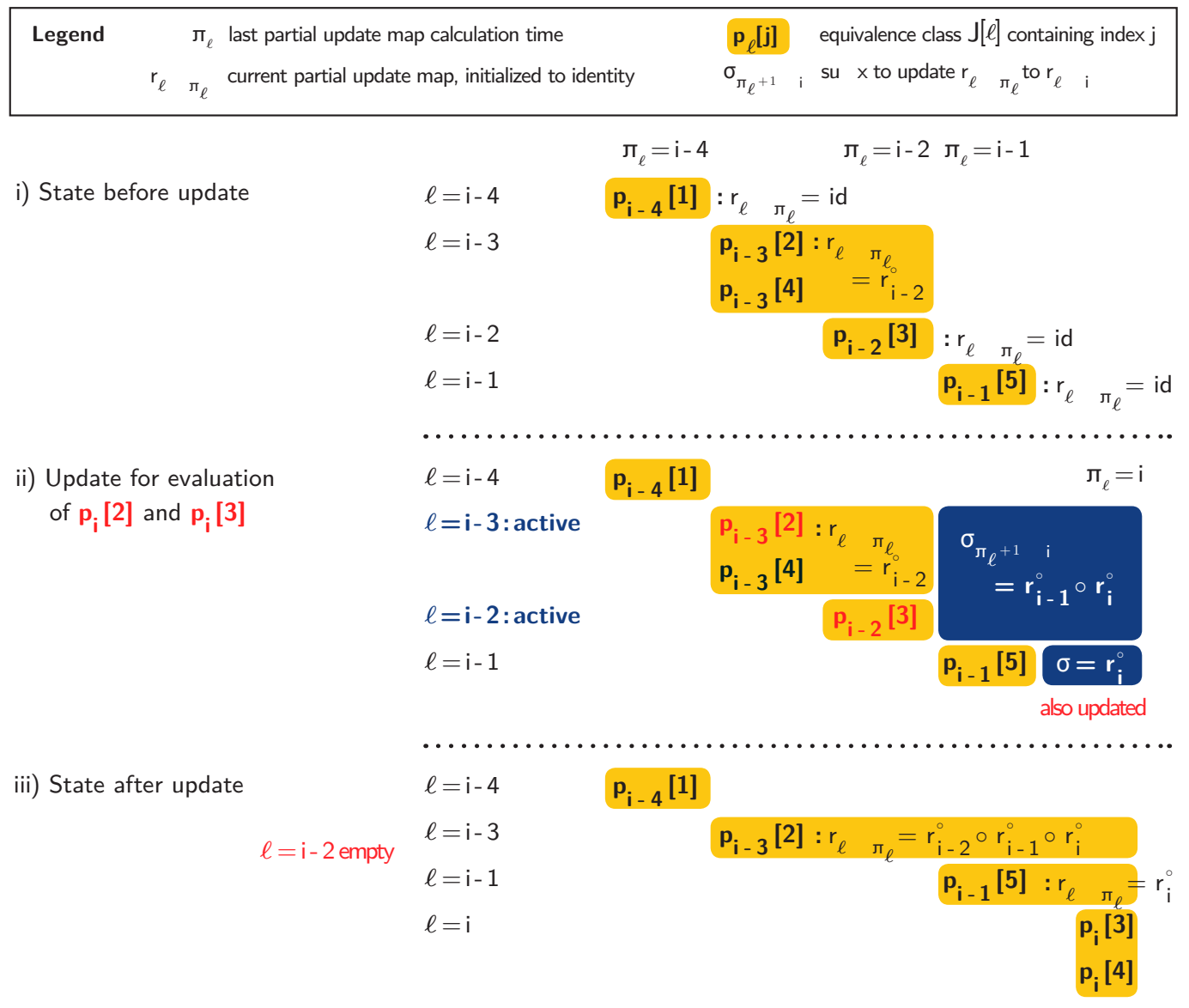

Figure 3 An illustration of the lazy evaluation states defined above as well as the steps of Algorithm 3. In this case, we have a lazy evaluation state at $\mathrm{i}=5$, which is updated with $\phi_{i}\left(o_{i}\right)=\{2,4\} . j=3$ is updated as well as specified by the first loop in the algorithm.

genetic site of both across a range of haplotype panel sizes from 30 to 5008 . Figure 5 shows this comparison. Observed time complexity of our algorithm was $\mathcal{O}\left(k^{0.35}\right)$ as calculated from the slope of the line of best fit to a log-log plot of time per site versus haplotype panel size.

For data points where we used all 1000 Genomes project haplotypes $(k=5008)$, on average, time per site is $37 \mu s$ for our algorithm and $1308 \mu s$ for the linear LS algorithm. For the forthcoming 100,000 Genomes Project, these numbers can be extrapolated to $251 \mu \mathrm{s}$ for our algorithm and $260,760 \mu \mathrm{s}$ for the linear LS algorithm.

\subsubsection{Lazy evaluation of dynamic programming rows}

We also measured the time which our algorithm spent within its lazy evaluation subalgorithm. In the average case, the time complexity of our lazy evaluation subalgorithm does not contribute to the overall algebraic time complexity of the algorithm. (Fig. 6, right) The lazy evaluation runtime also contributes minimally to the total actual runtime of our algorithm. (Fig. 6, left) 

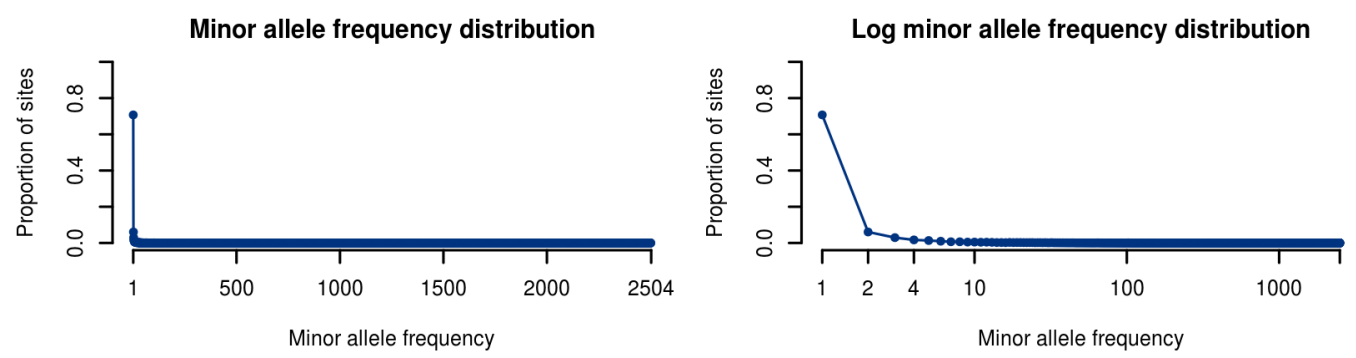

Figure 4 Biallelic site minor allele frequency distribution from 1000 Genomes chromosome 22.
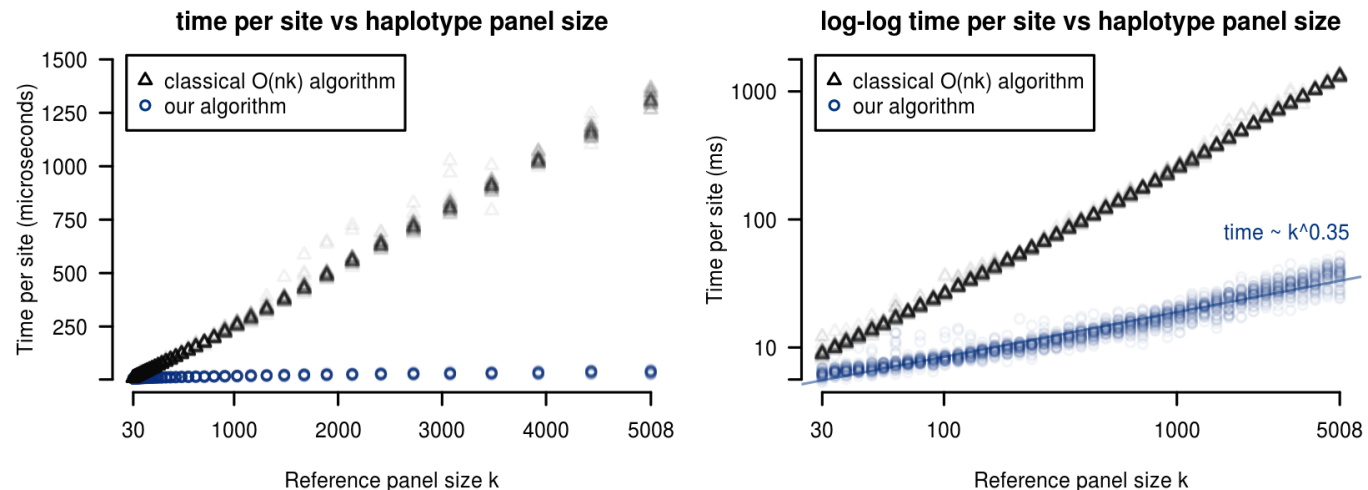

Figure 5 Runtime per site as a function of haplotype reference panel size $\mathrm{k}$ for our algorithm (blue) as compared to the classical linear time algorithm (black).

\subsection{Sparse haplotype encoding}

\subsubsection{Generating our sparse vectors}

We generated the haplotype panel data structures from ( $\$ 2)$ using the vcf-encoding tool vcf2slls which we provide. We built indices with multiallelic sites, which increases their time and memory profile relative to the results in (\$5.2) but allows direct comparison to vcf records. Encoding of chromosome 22 was completed in 38 minutes on a single CPU core. Use of $M \mathrm{CPU}$ cores will reduce runtime proportional to $M$.

\subsubsection{Size of sparse haplotype index}

In uncompressed form, our whole genome *.slls index for chromosome 22 of the 1000 genomes dataset was $285 \mathrm{MB}$ in size versus $11 \mathrm{~GB}$ for the vcf record using uint16_t's to encode haplotype ranks. When compressed with gzip, the same index was $67 \mathrm{MB}$ in size versus $205 \mathrm{MB}$ for the vcf record.

In the interest of speed (both for our algorithm and the $\mathcal{O}(n k)$ algorithm) our experiments loaded entire chromosome sparse matrices into memory and stored haplotype indices as uint64_t's. This requires on the order of 1 GB memory for chromosome 22. For long chromosomes or larger reference panels on low memory machines, algorithm can operate on sequential chunks of the reference panel. 

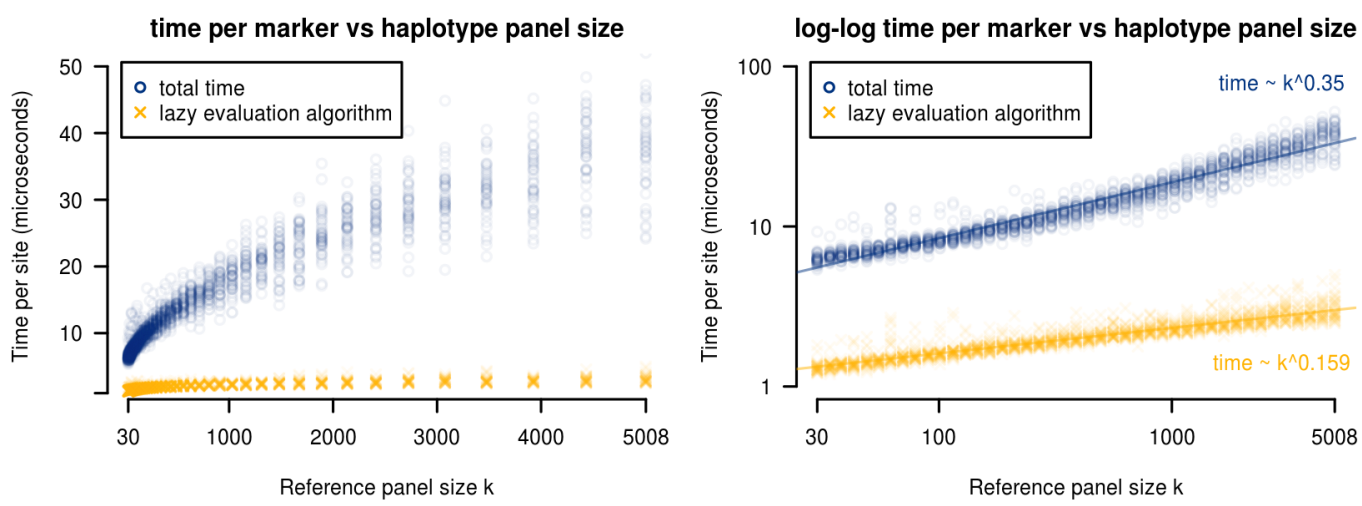

Figure 6 Time per site for the lazy evaluation subalgorithm (yellow) vs. the full algorithm (blue).

\section{Discussion and significance}

To the best of our knowledge, ours is the first forward algorithm for any haplotype model to attain sublinear time complexity with respect to reference panel size. Our algorithms could be incorporated into haplotype inference strategies by interfacing with our $\mathrm{C}++$ library. This opens the potential for tools which are tractable on haplotype reference panels at the scale of current 100,000 to $1,000,000+$ sample sequencing projects.

\subsection{Applications which use individual forward probabilities}

Our algorithm attains its runtime specifically for the problem of calculating the single overall probability $P(o \mid H, \rho, \mu)$ and does not compute all $n k$ forward probabilities. We can prove that if $m$ many specific forward probabilities are also required as output, and if the time complexity of our algorithm is $\mathcal{O}\left(\sum_{i}\left|\phi_{i}\right|\right)$, then the time complexity of the algorithm which also returns the $m$ forward probabilities is $\mathcal{O}\left(\sum_{i}\left|\phi_{i}\right|+m\right)$.

In general, haplotype phasing or genotype imputation tools use stochastic traceback or other similar sampling algorithms. The standard algorithm for stochastic traceback samples states from the full posterior distribution and therefore requires all forward probabilities. The algorithm output and lower bound of its speed is therefore $\mathcal{O}(n k)$. The same is true for many applications of the forward-backward algorithm.

There are two possible approaches which might allow runtime sublinear in $k$ for these applications. Using stochastic traceback as an example, first is to devise an $\mathcal{O}(f(m))$ sampling algorithm which uses $m=g(k)$ forward probabilities such that $\mathcal{O}(f \circ g(k))<\mathcal{O}(k)$. The second is to succinctly represent forward probabilities such that nested sums of the $n k$ forward probabilities can be queried from $\mathcal{O}(\phi)<\mathcal{O}(n k)$ data. This should be possible, perhaps using the positional Burrows-Wheeler transform [5] as in [11], since we have already devised a forward algorithm with this property for a different model in [13].

\subsection{Generalizability of algorithm}

The optimizations which we have made are not strictly specific to the monoploid Li and Stephens algorithm. Necessary conditions for our reduction in the time complexity of the recurrence relations are

- Condition 1. The number of distinct transition probabilities is bounded.

- Condition 2. The number of distinct emission probabilities is bounded. 
Favourable conditions for efficient time complexity of the lazy evaluation algorithm are

- Condition 1. The number of unique update maps added per step is bounded.

- Condition 2. The update map extension operation is composition of matrices of bounded size. This can be generalized to a broad algebraic class ${ }^{5}$ of update operations provided that they have bounded runtime.

The reduction in time complexity of the recurrence relations depends on the Markov property, however we hypothesize that the delayed evaluation needs only the semi-Markov property.

\subsubsection{Other haplotype forward algorithms}

Our optimizations are of immediate interest for other haplotype copying models. The following related algorithms have been explored without implementation.

- Example (Diploid Li and Stephens). We have yet to implement this model but expect average runtime at least subquadratic in reference panel size $k$. We build on the statement of the model and its optimizations in [9]. We have found the following recurrences which we believe will work when combined with a system of lazy evaluation algorithms:

- Lemma 11. The diploid Li and Stephens HMM may be expressed using recurrences of the form

$$
p_{i}\left[j_{1}, j_{2}\right]=\alpha_{p} p_{i-1}\left[j_{1}, j_{2}\right]+\beta_{p}\left(S_{i-1}\left(j_{1}\right)+S_{i-1}\left(j_{2}\right)\right)+\gamma_{p} S
$$

which use on the intermediate sums

$$
\begin{aligned}
S_{i} & :=\alpha_{c} S_{i-1}+\beta_{c} \sum_{j \in \phi_{i}} S_{i-1}(j)+\gamma_{c} \sum_{\left(j_{1}, j_{2}\right) \in \phi_{i}^{2}} p_{i-1}\left[j_{1}, j_{2}\right] & \mathcal{O}\left(\left|\phi_{i}\right|^{2}\right) \\
S_{i}(j) & :=\alpha_{c} S_{i-1}+\beta_{c} S_{i-1}(j)+\gamma_{c} \sum_{j_{2} \in \phi_{i}} p_{i-1}\left[j, j_{2}\right] & \mathcal{O}\left(\left|\phi_{i}\right|^{2}\right)
\end{aligned}
$$

where $\alpha_{(\cdot)}, \beta_{(\cdot)}, \gamma_{(\cdot)}$ depend only on the diploid genotype $o_{i}$.

Implementing and verifying this extension of our algorithm will be among our next steps.

- Example (Multipopulation Li and Stephens). [4] We maintain separate sparse haplotype panel representations $\phi_{i}^{A}\left(o_{i}\right)$ and $\phi_{i}^{B}\left(o_{i}\right)$ and separate lazy evaluation mechanisms for the two populations $A$ and $B$. Expected runtime guarantees are similar.

This model, and versions for $>2$ populations, will be important in large sequencing cohorts (such as NHLBI TOPMed) where assuming a single related population is unrealistic.

- Example (More detailed mutation model). It may also be desirable to model distinct mutation probabilities for different pairs of alleles at multiallelic sites. Runtime is worse than the biallelic model but remains average case sublinear.

- Example (Sequence graph Li and Stephens analogue). In [13] we described a hidden Markov model for a haplotype-copying with recombination but not mutation in the context of sequence graphs. Assuming we can decompose our graph into nested sites then we can achieve a fast forward algorithm with mutation.

- Example (Semi-Markovian recombination model). The lazy evaluation algorithm 3 may efficiently allow time-since-recombination dependent transition probabilities.

${ }^{5}$ Specifically, any collection of operations forming a category in the sense of category theory 


\section{References}

1 Brian L Browning and Sharon R Browning. A unified approach to genotype imputation and haplotype-phase inference for large data sets of trios and unrelated individuals. The American Journal of Human Genetics, 84(2):210-223, 2009.

21000 Genomes Project Consortium et al. A global reference for human genetic variation. Nature, 526(7571):68, 2015.

3 Olivier Delaneau, Jean-Francois Zagury, and Jonathan Marchini. Improved wholechromosome phasing for disease and population genetic studies. Nature methods, 10(1):5, 2013.

4 Peter Donnelly and Stephen Leslie. The coalescent and its descendants. arXiv preprint arXiv:1006.1514, 2010.

5 Richard Durbin. Efficient haplotype matching and storage using the positional burrowswheeler transform (pbwt). Bioinformatics, 30(9):1266-1272, 2014.

6 Alon Keinan and Andrew G Clark. Recent explosive human population growth has resulted in an excess of rare genetic variants. science, 336(6082):740-743, 2012.

7 John Frank Charles Kingman. The coalescent. Stochastic processes and their applications, 13(3):235-248, 1982.

$8 \mathrm{Na} \mathrm{Li}$ and Matthew Stephens. Modeling linkage disequilibrium and identifying recombination hotspots using single-nucleotide polymorphism data. Genetics, 165(4):2213-2233, 2003.

9 Yun Li, Cristen J Willer, Jun Ding, Paul Scheet, and Gonçalo R Abecasis. Mach: using sequence and genotype data to estimate haplotypes and unobserved genotypes. Genetic epidemiology, 34(8):816-834, 2010.

10 Po-Ru Loh, Petr Danecek, Pier Francesco Palamara, Christian Fuchsberger, Yakir A Reshef, Hilary K Finucane, Sebastian Schoenherr, Lukas Forer, Shane McCarthy, Goncalo R Abecasis, et al. Reference-based phasing using the haplotype reference consortium panel. Nature genetics, 48(11):1443, 2016.

11 Gerton Lunter. Fast haplotype matching in very large cohorts using the li and stephens model. bioRxiv, 2016. doi:10.1101/048280.

12 Jared O'Connell, Kevin Sharp, Nick Shrine, Louise Wain, Ian Hall, Martin Tobin, JeanFrancois Zagury, Olivier Delaneau, and Jonathan Marchini. Haplotype estimation for biobank-scale data sets. Nature genetics, 48(7):817, 2016.

13 Yohei Rosen, Jordan Eizenga, and Benedict Paten. Modelling haplotypes with respect to reference cohort variation graphs. Bioinformatics, 33(14):i118-i123, 2017.

14 Amy L Williams, Nick Patterson, Joseph Glessner, Hakon Hakonarson, and David Reich. Phasing of many thousands of genotyped samples. The American Journal of Human Genetics, 91(2):238-251, 2012. 